\title{
STEAM: a fast compact thermal model for two-phase cooling of integrated circuits
}

\author{
Arvind Sridhar ${ }^{1}$, Yassir Madhour ${ }^{1,2}$, David Atienza ${ }^{1}$, Thomas Brunschwiler ${ }^{2}$, John Thome ${ }^{1}$ \\ ${ }^{1}$ École Polytechnique Fédérale de Lausanne (EPFL), Switzerland \\ ${ }^{2}$ IBM Research Laboratory, Rüschlikon, Switzerland \\ \{arvind.sridhar, david.atienza, john.thome\}@epfl.ch, \{yma,tbr\}@zurich.ibm.com
}

\begin{abstract}
Two-phase liquid cooling of computer chips via microchannels etched directly on silicon dies is a potential long-term solution to enable continued integration of high-performance multiprocessors. Two-phase cooling refers to the heat removal via evaporation of a refrigerant flowing inside a heat sink. While possessing superior cooling properties, largescale use of this technology in the industry is limited by the lack of thermal modeling tools that can accurately predict temperatures in a two-phase cooled IC. In this paper, we propose STEAM, a new compact thermal model for $2 \mathrm{D} / 3 \mathrm{D}$ ICs with two-phase cooling via silicon microchannels. The accuracy of the STEAM model is validated against measurements from a real two-phase cooled IC test stack reported previously in literature. Temperatures were predicted with an average error as low as $10.2 \%$ for uniform heat fluxes and $6.9 \%$ for hotspots. Finally, the STEAM model is applied to a realistic $3 \mathrm{D}$ multiprocessor system-on-chip (3D MPSoC) with two-phase cooling to simulate IC temperatures and the refrigerant pumping power, demonstrating the applicability of STEAM in the early-stage design of near-future high-performance computers with two-phase cooling.
\end{abstract}

\section{Categories and Subject Descriptors}

1.4 [SYSTEM-LEVEL CAD]: [Power and Thermal Considerations in System Design]

\section{Keywords}

Two-phase cooling, Thermal modeling, 3D ICs

\section{INTRODUCTION}

Various advanced thermal packages have been proposed in the recent years to combat the rising heat fluxes of high-

\footnotetext{
* This work has been partially supported by the Nano-Tera RTD project CMOSAIC (ref.123618), financed by the Swiss Confederation and scientifically evaluated by SNSF, and the EC FP7 GreenDataNet STREP project (Agreement No. 609000).
}

performance computer chips that the conventional air-cooled heat sinks can no more handle [1]. In particular, two kinds of liquid cooling via microchannels, etched directly on the surface of silicon dies, are being explored: single-phase cooling using water and two-phase cooling using industrial refrigerants [2]. Single-phase cooling refers to the heat removal by a coolant that remains in liquid state and rises in temperature inside the heat sink (sensible heat absorption). Several prototype 2D and 3D ICs have been built to demonstrate that single-phase liquid cooling has superior cooling capabilities compared to air cooling $[3,4]$.

Two-phase cooling refers to heat removal by the evaporation of a refrigerant in the heat sink. Here, the heated surface is cooled via the latent heat absorption, i.e., the conversion of the physical state from liquid to vapor. Generally, latent heat absorption of fluids results in a far higher heat removal than sensible heat absorption [5]. Hence, theoretically, two-phase cooling can be far more efficient than singlephase cooling of ICs. In addition, because refrigerants remain at an almost constant temperature (ideally) when boiling, two-phase cooling naturally overcomes the problem of induced thermal gradients encountered in single-phase cooling [6]. These principles have been practically demonstrated using prototypes of two-phase liquid cooled ICs built by various research groups $[2,7]$. Moreover, two-phase cooling is already being used is some power electronics applications [8].

In spite of these obvious advantages, adoption of the twophase cooling technology by the industry is limited partly because of the lack of widely available of computer-aided design (CAD) tools that can enable the energy-efficient and temperature-aware design of such devices. The following are the challenges to the development of CAD tools for twophase cooling:

1. Two-phase heat transfer, especially at the micrometer scale, is an extremely complex phenomenon to accurately model; conventional fine-grained simulation softwares for two-phase flows are still in the development stage.

2. Due to the complexities of computational modeling of two-phase flows, most existing studies have focused on building experimental prototypes of two-phase heat exchangers and developing measurements-based empirical correlations for the relationships between the various parameters of heat transfer such as temperature, heat flux, mass flow rate, pressure etc.

3. Integrated two-phase cooling for ICs as a concept is fairly new in the area of IC design. The knowledge of two-phase flows and heat transfer is largely contained in the thermal engineering literature, and the methods developed there 
are incompatible with the other existing CAD tools for electronics design.

In this paper, we propose a new thermal model called STEAM ("Simulation of Two-phase Energy And Mass balance"), a new compact thermal model for two-phase cooling of $2 \mathrm{D} / 3 \mathrm{D}$ ICs that models the complex problem of conjugate heat conduction-convection in a two-phase cooled IC. The main contributions of the paper are as follows:

1. The fundamentals of two-phase cooling using microchannels are described and a brief literature review is presented from the perspective of early-stage design of ICs.

2. A compact mathematical model for the heat transfer (energy balance) and evaporation of flowing liquid (mass balance) called STEAM is derived from first principles. The STEAM model is flexible, enabling the easy implementation of any of the different studies in the literature on two-phase heat transfer.

3. Accuracy of the STEAM model is validated against measurements from a real two-phase cooled IC test stack. Results indicate that the STEAM can simulate temperatures with average errors as low as $10.2 \%$ (where typical errors in literature are much higher).

4. The STEAM model is then used to perform temperature and pumping power simulations of a realistic 3D MPSoC with two-phase cooling- demonstrating the efficacy of STEAM as a tool for early-stage design of $2 \mathrm{D} / 3 \mathrm{D}$ ICs with two-phase cooling.

The rest of the paper is organized as follows. Section 2 describes the fundamentals of two-phase flows and heat transfer and provides a brief review of existing literature on this topic. Section 3 describes the target problem being modeled, Section 4 presents the derivation of the proposed STEAM model and the implementation details andSection 5 describes the experimental validation of the accuracy of STEAM against measurement data. In Section 6, we analyze a realistic twophase cooled 3D MPSoC with STEAM and, finally, Section 7 summarizes the main conclusions.

\section{TWO-PHASE COOLING}

Two-phase flows on a macro-scale have been an integral part of various industries (refrigeration, energy, etc.) for many decades. Recently, owing to their growing importance in tackling rising heat fluxes in high-performance computers [9], the study of micro-scale two-phase flows has also gained prominence. Various research groups have built prototypes of two-phase cooled ICs (see [1] for a complete review). The modeling of two-phase cooling begins with the study of the nature of two-phase flows.

Fig. 1(a) provides an illustration of the basic processes taking place inside a small section of micrometer scale rectangular channel with two-phase cooling. A refrigerant flows from left to right, absorbing the heat entering via the channel surfaces. In the process, the refrigerant evaporates incrementally, resulting in an increase in the vapor qualitydefined as the fraction of the vapor mass flow rate with respect to the total mass flow rate. The nature of the boiling process of the refrigerant, combined with the nature of its flow down the channel generates various regimes of flow such as bubbly flow, stratified flow, slug flow, etc. that can be analyzed using visualization studies [10]. Some relevant flow

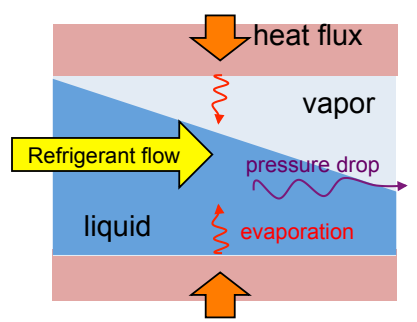

(a)

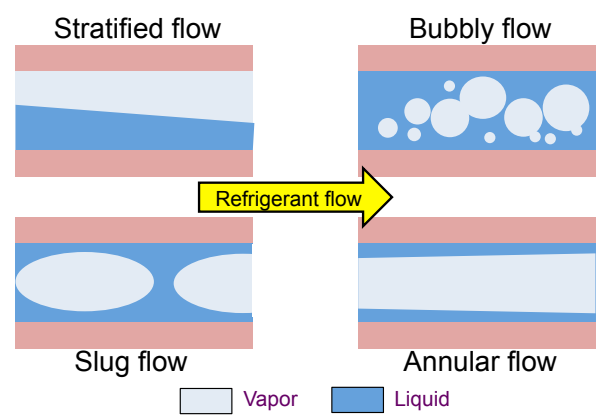

(b)

Figure 1: (a) Fundamentals of two-phase cooling (b) Examples of two-phase flow regimes.

regimes are shown in Fig. 1(b). These flow regimes determine how the liquid and the vapor phases interact with each other in the channel. The specific flow regime encountered in a particular case depends upon various factors such as the physical properties of the refrigerant, flow velocity and experimental conditions.

Thus, the local heat removal capability of the two-phase flow at a particular point along the channel (i.e., the cooling efficiency) is a complex function of:

1. Flow regime of the two-phase flow.

2. Thermal conductivities of the liquid and vapor phases.

3. The local mass fraction (vapor quality) and the volume fraction (called void fraction) of the vapor with respect to the liquid phase.

4. The proportions of the channel surface area wetted by the liquid phase and "dried" by the vapor phase, and the local thicknesses of the liquid/vapor films.

5. The nature of the flow within the individual phases (i.e. laminar or turbulent or transitionary).

6. The local temperature, pressure and the local heat flux entering the channel.

The modeling problem is further complicated by the fact that in two-phase conditions, the temperature is a strict function of pressure [11]. This means that local saturation pressures of the refrigerant inside the microchannels must be first computed in order to calculate the temperatures of the refrigerant and, in turn, the entire IC. Consequently, the development of conventional numerical methods such as finite-element simulations are still in the early stages for two-phase cooling [12].

Hence, traditionally, studies on two-phase cooling are focussed on extensive experiments performed on prototype devices, and studying the relationship between various experimental conditions and heat transfer properties of two-phase flows. An empirical fitting of the results is used to determine 
the complex relationships between the parameters described above. A rich body of literature exists in the domain of thermal engineering based on these studies. Due to the lack of space, it is impossible to review all these works in this paper. The reader is instead referred to Chapter 20 in [13] for a comprehensive survey of the research in this topic. The following are the main drawbacks of these studies, with respect to temperature-aware design of ICs:

1. The goal for these studies is the evaluation/prediction heat transfer coefficients (HTCs, or cooling efficiencies) and pressure drops for micro-scale two phase flows and not to develop a comprehensive CAD tool for early-stage design space explorations for ICs with two-phase cooling, which is the main concern for IC designers.

2. Most of these studies rely on uniform heat flux distributions (except a few such as [14]) in their experiments and evaluate HTCs using 1D or simplified 3D heat conduction models for the solid structures in the IC. In a realistic IC, such as a multi-core processor, heat fluxes are highly non-uniform owing to the heterogenous nature and functioning of different logic elements [15]. Hence, a fully coupled three-dimensional conduction-convection model for the entire IC becomes necessary for the accurate calculation of temperatures.

3. Many of these studies provide HTC prediction methods based on the experimental data only from that particular study, and hence are susceptible to the unique experimental conditions and errors that cannot be replicated elsewhere. Typically, different methods show strong disagreements in their relative accuracies with respect to a particular set of measurements. Errors in some cases can be very high $(\sim 50 \%)$.

Compact thermal models such as HotSpot [16] and 3DICE [17] have been proposed specifically for temperatureaware design for air-cooled and single-phase liquid cooled ICs respectively. A similar method for early-stage design space explorations (floorplan evaluations, combined HWSW cosimulation etc.) is needed for two-phase cooling. In this paper, we utilize the knowledge available in the thermal engineering domain, and propose the STEAM model in order to address the above challenges. In particular, STEAM provides a simple, compact and flexible simulation framework for two-phase cooled ICs, where it is possible to incorporate HTCs from any previous study of two-phase flows. This capability is demonstrated in this work using three different methods from the literature.

\section{TARGET IC ARCHITECTURE}

The proposed STEAM thermal model is versatile in simulating a $2 \mathrm{D}$ or even a $3 \mathrm{D}$ IC with two-phase cooling via microchannels. For the presentation of the model, we choose a concept IC with two-phase cooling, based on a test structure built and studied by the authors of $[14,18]$, illustrated in Fig. 2(a). It consists of a single $(12.7 \mathrm{~mm} \times 17.78 \mathrm{~mm})$ silicon die. 35 resistive heaters in a $5 \times 7$ grid on the surface of this die mimic the different heat dissipating logic blocks of a large microprocessor, such as the 48-core Intel Single-Chip Cloud Computer (Fig. 2(b)) [19].

Microchannels were etched on a silicon substrate and attached to the silicon die via a high conductance thermal interface layer. Finally, the top surface of the structure

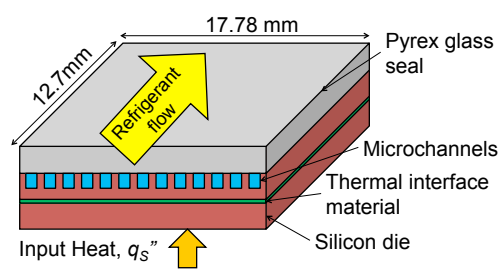

(a)

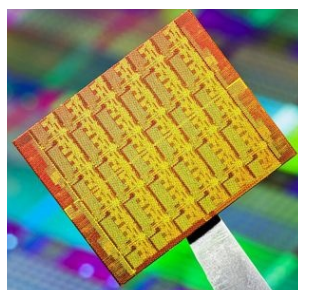

(b)
Figure 2: (a) Target architecture of the IC with two-phase cooling via silicon microchannels (b) Intel Single-Chip Cloud Computer [19].

(with the channels) is hermetically sealed using an insulating pyrex glass. There are a total of 135 microchannels of crosssectional dimensions $85 \mu \mathrm{m} \times 560 \mu \mathrm{m}$ with $45 \mu \mathrm{m}$ thick silicon walls between the channels. These microchannels were injected with industrial refrigerants such as R-236fa or R$245 \mathrm{fa}$. For further details about the structural and material properties of the test structure, please refer to Section 5.

At any point along the microchannel, the physical state of the refrigerant can be uniquely described using three parameters: pressure, temperature and vapor quality. Without loss of generality, we begin with the following assumptions:

1. The two-phase cooled microchannel heat sink is the only path for heat dissipation in the system, with zero heat escaping via other exposed surfaces.

2. The refrigerant is assumed to be already at saturation conditions at the inlet (i.e, evaporating or just starting to evaporate), and there is no sensible heat absorption in the refrigerant. Given the relatively low pressure (or temperature) drops seen in microchannel heat sinks [1], this is a reasonable assumption.

3. The inlet physical state of the refrigerant is known.

Our goal is to simulate the three-dimensional distribution of temperatures in the IC and the physical state of the refrigerant inside the microchannels.

\section{THERMAL MODELING IN STEAM}

In this section our proposed modeling approach in STEAM will be presented. For a full list of symbols used in the ensuing sections and their meanings, please refer to the section on "Nomenclature" at the end of the paper.

Compact thermal modeling in STEAM begins with discretizing the target computational domain into small "thermal cells"- small cuboidal blocks- such that each cell contains only one type of material. Such a discretization for the target computational domain in Fig. 2(a) is illustrated in Fig. 3. We now have "solid thermal cells" and "microchannel thermal cells" consisting of the refrigerant flowing under two-phase conditions, as shown in Fig. 4. Each type of thermal cell must be modeled differently .

\subsection{Compact modeling for solids}

For the solid thermal cells, the conventional compact thermal modeling for heat conduction in solids is applied [16]. This is illustrated for a single solid cell in Fig 4(a). In this representation, each solid cell is converted into an equivalent electrical circuit where the voltage represents temperature $T$ of the cell and current represents heat flow. The heat conduction in all the six directions of the cartesian coordinate 


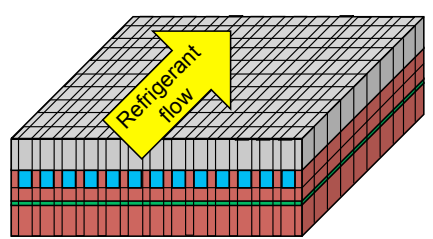

Figure 3: Target computational domain in Fig. 2(a) discretized into thermal cells for compact modeling in STEAM.
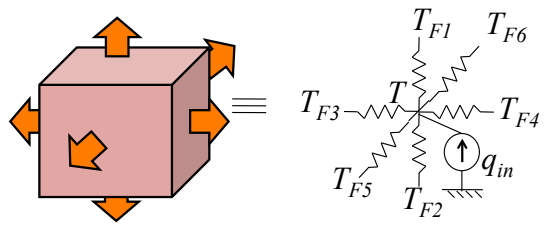

(a)

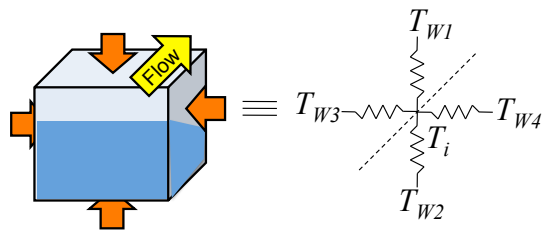

(b)

Figure 4: (a) Solid thermal cell (b) Two-phase thermal cell.

system are represented by thermal conductances (or resistances) $g_{1-6}$. When there is heat generation in the solid cell because of electrical switching activity in the IC, it is represented using a input current source term $q_{i n}$ applied directly to the cell as shown in Fig. 4(a).

Thus, the steady state energy balance equation (i.e. the process of equating total heat entering with the total heat leaving the cell) inside the solid thermal cell can be written for this equivalent electrical circuit as:

$$
\sum_{k=1}^{6} g_{k}\left(T-T_{F k}\right)=q_{i n},
$$

where $T_{F 1-6}$ are the temperature of the 6 faces of the thermal cell. The conductances $g_{1-6}$ can be calculated using the thermal conductivity of the solid material constituting the cell and the cell dimensions. Neighboring cells can be connected via the cell interfaces and the complete 3D heat conduction model can be constructed in the form of a resistive grid circuit [16].

\subsection{Energy and mass balance for two-phase flows}

A two-phase thermal cell is illustrated in Fig. 4(b), along with the flow direction and the microchannel walls from which heat enters the refrigerant. The complexities of heat transfer from the walls into the refrigerant (as described in Section 2 and 3) are contained by raising the level of abstraction in the STEAM model. This is accomplished using a 4-conductance model (or 4-resistor model) to represent the heat entering via convection from the four microchannel walls $(W 1-4)$ into the refrigerant bulk as shown in Fig 4(b). To derive the values of these conductances and to

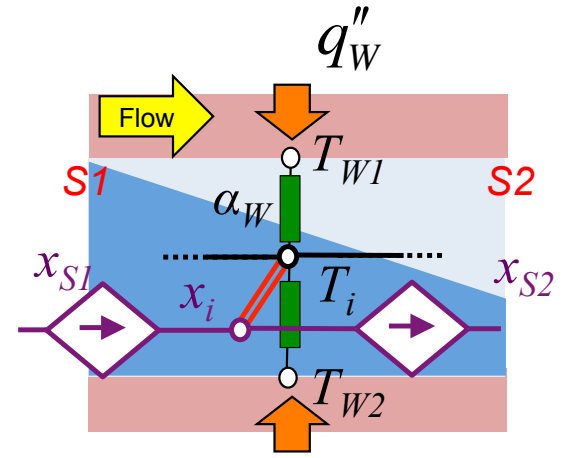

Figure 5: Compact thermal model of a two-phase thermal cell in STEAM.

evaluate the evolution of temperature along the direction of flow inside the microchannel, the energy and mass balance equations inside each two-phase thermal cell must be solved for, as described below.

Consider the side view of the $i^{\text {th }}$ two-phase thermal cell (counting from the inlet along the microchannel) illustrated in Fig. 5. Here, the direction of the flow is from left to right, and the convective resistances to the side walls $(W 3-4)$ are not shown. This cell borders the $(i-1)^{t h}$ and $(i+1)^{t h}$ thermal cells along the front and rear interfaces S1 and S2 respectively. The heat transfer in this thermal cell can be expressed using the steady state energy balance equation as follows:

$$
\begin{aligned}
& \text { Heat entering from }(i-1)^{t h} \text { cell via surface } \mathrm{S} 1 \\
& + \text { Heat entering from the microchannel walls } \\
& =\text { Heat exiting to the }(i+1)^{t h} \text { cell via surface } \mathrm{S} 2
\end{aligned}
$$

In two-phase cooling, the first and the last terms in the equation above are contributed by the flow of the refrigerant and the corresponding flow of enthalpy, or thermal energy, down the channel. The thermal energy of a refrigerant under two-phase conditions is dominated by the vapor quality [11]. That is, thermal energy entering from the walls of the microchannel is stored as the conversion of liquid into vapor as illustrated in Fig. 1(a). The rate of flow of enthalpy, thus, is directly given by the function $h_{l v} \dot{M} x$. The second term is a function of the difference between the local wall temperatures $T_{W 1-4}$ and the local bulk temperature of the refrigerant $T_{i}$, and the heat transfer coefficient, $\alpha_{W}$ at the wall surface, which is defined as the rate of flow of heat per unit area per kelvin of temperature difference. Thus, the energy balance in $\mathrm{Eq}(2)$ can be written as:

$$
\begin{aligned}
& h_{l v} \dot{M} x_{S 1}+\sum_{k=1}^{4} \alpha_{W}\left(T_{W k}-T_{i}\right)=h_{l v} \dot{M} x_{S 2}, \\
& \Rightarrow h_{l v} \dot{M}\left(x_{S 2}-x_{S 1}\right)+\sum_{k=1}^{4} \alpha_{W} T_{W k}=4 \alpha_{W} T_{i},
\end{aligned}
$$

where $x_{S 1}$ and $x_{S 2}$ are the vapor qualities of the refrigerant at the two interfaces of the thermal cell. The following observations can be made about the above equation:

1. The above equation provides a compact representation for heat transfer processes in two-phase flows. This is done by encapsulating the complexities of the two-phase heat transfer processes into a single parameter: the heat transfer coefficient $\alpha_{W}$. 
2. Note that the mass balance equations for the vapor quality are implicitly coupled with the above energy balance equations. That is, when solving for the temperatures, the vapor quality distribution of the refrigerant along the microchannels are also automatically solved for. This coupling of the energy and mass balances is illustrated in Fig. 5 where the circuit drawn in violet represents mass balance and the circuit drawn in black represents the energy balance.

3. As before, the equivalent circuit shown in Fig. 4(b) can be connected to the neighboring 4 solid cells (Fig. 4(a)) via the cell interfaces. Thus, a complete $3 \mathrm{D}$ thermal circuit grid can be created that models the conjugate $3 \mathrm{D}$ conduction and convection in the two-phase cooled IC.

4. In the above equations the refrigerant temperature $T_{i}$ is assumed to be already known and is put on the righthand side as an input. In Section 4.2.2, the method for calculating this temperature is explained.

5. The inlet and outlet vapor qualities for each two-phase cell $x_{S 1}$ and $x_{S 2}$ can be obtained from the cell vapor qualities $x_{i}$ using central-differencing.

Thus, the equations (1) and (3) can be combined to construct the final coupled STEAM model for two-phase cooling of ICs as follows:

$$
\mathbf{G X}=\mathbf{U},
$$

where $\mathbf{X}$ is the vector of temperatures in silicon and the vapor qualities inside the microchannel, $\mathbf{G}$ is the conductance matrix of the system, and $\mathbf{U}$ is the vector of sources $\left(q_{i n}\right)$ and boundary conditions (represented by $\left.T_{i}\right)$. Solving this circuit, thus, simultaneously yields the temperatures in the silicon structures and the vapor qualities inside the microchannels from inlet to outlet.

\subsubsection{Heat transfer coefficients for Two-Phase cooling}

As mentioned in Section 2, the local heat transfer coefficient $\alpha_{W}$ in $\mathrm{Eq}(3)$ is a complex function of various paramters. Thus, we use previous methods in the literature to compute this parameter for our purposes. The general agreement across many studies is that the most important parameters influencing the local HTC are the local heat flux from the walls $q_{W}^{\prime \prime}$ and the local vapor quality $x$ [13]. For example, Tran et al $[13,20]$ conducted experiments using several refrigerants and developed a statistical correlation for the local HTCs for micro-scale two-phase flows given by:

$$
\alpha_{W}=8.4 \times 10^{5}\left(B o^{2} W e_{l}\right)^{0.3}\left(\frac{\rho_{l}}{\rho_{v}}\right)^{-0.4} .
$$

Using this method the local HTCs can be computed and substituted in $\mathrm{Eq}(3)$. There are several other methods to compute HTCs- both simple and complex- that can be easily incorporated in STEAM. In this paper, we have incorporated and studied three such methods (Section 5).

\subsubsection{Pressure-drop in two-phase flows}

As mentioned in Section 4.2, an important parameter in $\mathrm{Eq}(3)$ that must be computed is the refrigerant temperature $T_{i}$. Ideally, under two-phase conditions, fluid temperature remains constant as it evaporates since all the heat that enters the microchannel is used for phase conversion. However, in reality, there is a slight decrease of temperature from inlet to the outlet. This is because (as described in Section 2) under two-phase conditions, temperature is strictly a function of pressure [11] and there is a drop in pressure from inlet to outlet of the microchannel. This pressure drop is due to change in fluid momentum of the fluid from inlet to the outlet as well as friction at the fluid-wall and liquidvapor interfaces [13]. Pressure drop is also a measure of the effort required to pump the refrigerant into the microchannels. Pumping power- defined as the product of the pressure drop and the inlet volumetric flow rate- is an important parameter in the temperature-aware design of liquid-cooled ICs.

Similar to the HTCs, there are various experiment-based methods available for predicting local pressure-drop in the literature. The reader is referred to [13] for a full list of possible pressure-drop correlations. As an example, according to the homogenous pressure-drop approach, the local momentum and frictional pressure drops at a distance $z$ from the inlet inside the microchannel are given by [13]:

$$
\left.\frac{d p}{d z}\right|_{\text {mom }}=\frac{d\left(G / \rho_{T P}\right)}{d z},\left.\frac{d p}{d z}\right|_{\text {frict }}=\frac{2 f_{T P} G^{2}}{d_{h} \rho_{T P}},
$$

where the two-phase friction factor $f_{T P}$ is calculated using the Blasius equation [13]. Hence, at the $i^{\text {th }}$ thermal cell, the pressure can be computed using the finite-difference approximation as follows:

$$
p_{i}=p_{i-1}-\Delta p_{i}=p_{i-1}-\left[\left.\frac{d p}{d z}\right|_{i, m o m}+\left.\frac{d p}{d z}\right|_{i, \text { frict }}\right] \Delta z_{i}
$$

Once the local pressures of the refrigerant are computed at all points along the microchannel, software databases for fluids, such as REFPROP [21], can be used to derive the corresponding temperatures, which are then substituted on the right-hand side in $\mathrm{Eq}(3)$ to build/update the STEAM thermal model.

\subsection{STEAM simulation cycle}

As can be seen from $\operatorname{Eq}(3-7)$, the local HTCs, the local physical state of the refrigerant and the solution to the thermal model are all strongly dependent on each other. Hence, we need an iterative solving methodology to correctly simulate the STEAM model. In this regard, our implementation of the STEAM simulator is illustrated in Fig. 6:

1. First, based on an initial guess of the refrigerant's physical state in the microchannels (eg. the inlet conditions), local HTCs are computed and substituted in $\mathrm{Eq}(3)$.

2. Next, these equations are combined with the heat conduction model of the IC in Eq(1) and the 3D STEAM equivalent circuit is generated. Thus, the matrices in $\mathrm{Eq}(4)$ are constructed.

3. $\mathrm{Eq}(4)$ is solved to obtain the IC temperatures $T$ and vapor qualities $x$ of the refrigerant.

4. Using the temperatures of the IC along the microchannel wall, the local wall heat fluxes entering the refrigerant $q_{W}$ are computed. These parameters are then given as input to $\mathrm{Eq}(5-7)$ to obtain the local HTCs $\alpha_{W}$ and local pressures of the refrigerant $p_{i}$.

5. Using the REFPROP software, the local temperatures of the refrigerant $T_{i}$ are computed. These values and the HTCs are substituted in $\mathrm{Eq}(3)$.

6. The matrices in $\mathrm{Eq}(4)$ are updated and we go back to step 3 . This process is repeated until convergence. 


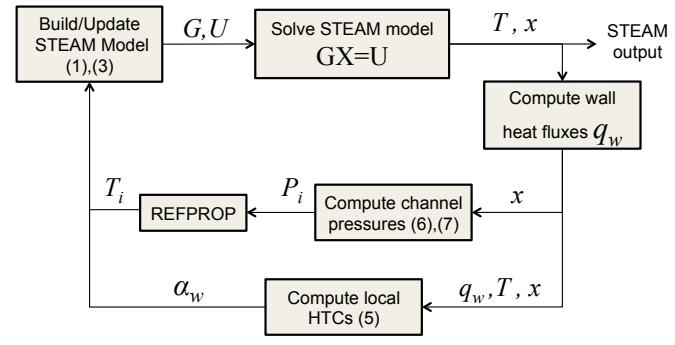

Figure 6: Simulation cycle of the STEAM model.

The entire STEAM model, along with this iterative routine, was implemented in Matlab. Eq(4) was solved using the sparse LU solver available in Matlab. The simulations were performed in a computer with Intel Corei7 processor (3.4 GHz) with $32 \mathrm{~GB}$ RAM. The REFPROP program [21] needed for the simulations was directly called from Matlab.

\section{ACCURACY VALIDATION OF STEAM}

In this section, we validate the accuracy of the STEAM model. To do this, we compare results from STEAM simulations to experimental measurements from actual two-phase cooled IC test sections that have been built and studied in the literature. In this study, we choose the work done by $[14,18]$. The silicon test structure was built in this study to simulate a real electronic IC, as illustrated in Fig. 2(a).

The floorplan on the surface of the silicon-die in the test structure is shown in Fig. 7. As described in Section 3, it contains 35 independent resistance heaters in a $5 \times 7$ grid numbered as rows and columns as shown in Fig. 7. The direction of the flow in the microchannels is from top to bottom of these figures. There is one temperature sensor at the center of each of these heater blocks giving temperature measurements at 35 locations. The structural properties of the test structure are tabulated in Table 1 . Next, the following two experiments were performed.

- Experiment 1- Uniform heat flux: In the first case, all the heaters were fired to a uniform heat flux density of $96.4 \mathrm{~W} / \mathrm{cm}^{2}$ (Fig $7(\mathrm{a})$ ). The refrigerant used in this case was R-236fa (Hexafluoropropane) at an inlet temperature of $31.1^{\circ} \mathrm{C}$ in a purely liquid state (Saturation pressure, $P_{\text {sat }}=$ $3.32 \mathrm{bar}$ ), and a mass flow velocity of $933 \mathrm{~kg} / \mathrm{m}^{2} \mathrm{~s}$.

In the STEAM model, the structure was divided using thermal cells of size $85 \mu \mathrm{m} \times 254 \mu \mathrm{m}$. The pressure drops inside the channels were calculated using the homogenous model described in Section 4.2.2. For the HTCs, in order to study the effect of using different methods from the literature in the STEAM model on the accuracy, three different methods were used as case studies: Bertsch et al. (2009) [22], Kandlikar et al. (2004) [23] and Tran et al. (1996) [20].

The simulations in all cases converged in 3-4 iterations taking a total time of about $35 \mathrm{sec}$. The results from the STEAM model were then compared with the experimental data for temperatures from the various sensors on the silicon surface. Sample temperature results for the sensors in col\#4 of Fig. 7 are plotted against simulation results as a function of the distance from inlet in Fig. 8(a). The results from STEAM are plotted using solid lines with different colors corresponding to the different correlations used to predict HTCs (Bertsch et al. in red, Kandlikar et al. in green and Tran et al. in blue). The discreet plot labelled "Data" refers to the measurement data. As can be seen, while STEAM is

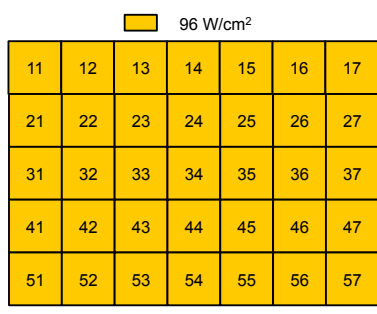

(a)

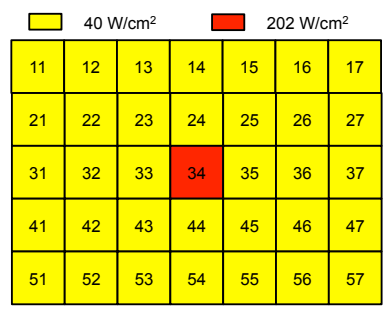

(b)
Figure 7: Heat flux patterns in the test structure for (a) Experiment 1 and (b) Experiment 2.

Table 1: Structural properties of the test structure $[14,18]$

\begin{tabular}{|l|l|}
\hline Individual heater size & $2540 \mu \mathrm{m}$ \\
\hline Channel width & $85 \mu \mathrm{m}$ \\
\hline Channel height & $560 \mu \mathrm{m}$ \\
\hline Number of channels & 135 \\
\hline Hydraulic diameter $D_{h}$ & $148 \mu \mathrm{m}$ \\
\hline Silicon die thickness & $350 \mu \mathrm{m}$ \\
\hline TIM thickness & $50 \mu \mathrm{m}$ \\
\hline Microchannel base thickness & $440 \mu \mathrm{m}$ \\
\hline
\end{tabular}

able to predict the general trend in the temperatures, some correlations fare better than the others.

In order to study the errors, the $\Delta T$ - defined as the difference between the silicon temperature and the inlet refrigerant temperature $\left(31.1^{\circ} \mathrm{C}\right)$ - at all the sensor locations from STEAM simulations were plotted along with the corresponding data from measurements in a scatter plot in Fig. 9. As can be seen here, while STEAM predicts temperatures in most cases within $+/-30 \%$, HTCs computed from Bertsch et al. [22] result in the most accurate predictions in this particular experiment (average error of $13.8 \%$ ). The average errors from each of these methods is tabulated in Table 2.

Note that the differences in the errors when using different HTC correlations can be attributed to the specific conditions of the experiments performed by the authors of these correlations (i.e. range of parameters such as flow rates, dimensions etc. explored in these studies) that may or may not conform to the conditions in our current experiment, as explained in Section 2. While from an EDA perspective, errors of $10.2 \%$ could be considered to be high, it must be noted that this is a favorable result for two-phase simulations considering existing literature on this subject [13], especially for early-stage design of ICs. Moreover, STEAM provides a very effective platform for architecture-level thermal simulation, where HTCs from any of the existing studies can be easily

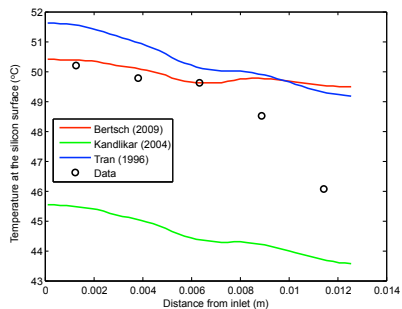

(a)

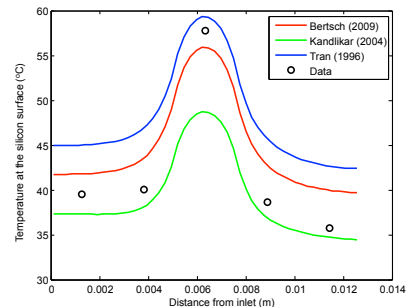

(b)
Figure 8: Comparison of temperatures from col\#4 (Fig. 7) between STEAM model and measurement data for (a) Experiment 1 and (b) Experiment 2. 


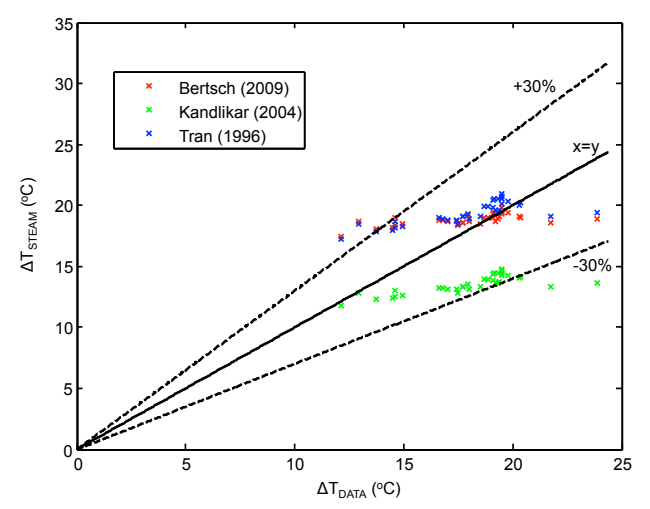

Figure 9: Scatter $\Delta T_{S T E A M}$ vs $\Delta T_{\text {data }}$ for various HTC correlations used in the STEAM model showing the spread of error in Experiment 1.

Table 2: Average errors incurred by STEAM model using various HTC correlations (Experiment 1)

\begin{tabular}{|c|c|}
\hline Correlation & Average Error (\%) \\
\hline Bertsch et al. (2009)[22] & $10.2 \%$ \\
\hline Kandlikarl et al. (2004)[23] & $23.7 \%$ \\
\hline Tran et al. (1996) [20] & $11.2 \%$ \\
\hline
\end{tabular}

incorporated based on the current design specifications, as has been demonstrated by this experiment.

- Experiment 2- Hotspot heat flux: In order to simulate the effects of non-uniform heat fluxes typically found in real microprocessors, in the second experiment, a single heater (at location row\# 3, col\# 4) was fired to a very high heat flux density $\left(200 \mathrm{~W} / \mathrm{cm}^{2}\right)$ while the rest of the heaters had a relatively low heat flux density $\left(40 \mathrm{~W} / \mathrm{cm}^{2}\right)$ as shown in Fig. 7(b). The refrigerant used in this experiment was R245fa (Pentafluoropropane), injected at a temperature of $31.1^{\circ} \mathrm{C}$ in a purely liquid state $\left(P_{\text {sat }}=1.84 \mathrm{bar}\right)$ with a mass flow velocity of $713 \mathrm{~kg} / \mathrm{m}^{2} \mathrm{~s}$.

The STEAM model was constructed and simulated similar to Experiment 1. In this case, the temperature comparison for col\# 4 is shown in Fig. 8(b). Again, it was found that the accuracy of the STEAM model depended strongly upon the particular correlation used to predict the HTCs (best-case hotspot error of $6.9 \%$ ).

\section{SIMULATION OF A REALISTIC 3D MPSOC}

In this section, we apply the STEAM model to a realistic 3D MPSoC to demonstrate the efficacy of STEAM as a tool for early-stage design of multiprocessor architectures with two-phase cooling. For this, we consider the $1.1 \mathrm{~cm} \times 1.1 \mathrm{~cm} 2$ die version of the 8-core UltraSPARC T1 Niagara processor architecture proposed by the authors of [15]. The 2-dies (one with cores the other with cache memories) sandwich a single two-phase microchannel cavity. The structure and the floorplan of this architecture are shown in Fig. 10.

The refrigerant used in this simulation was R-245fa, injected at a temperature of $31.1^{\circ} \mathrm{C}$ in a purely liquid state $\left(P_{\text {sat }}=1.84 \mathrm{bar}\right)$ with a mass flow velocity of $700 \mathrm{~kg} / \mathrm{m}^{2} \mathrm{~s}$. The STEAM model for this structure was implemented using the Bertsch et al. (2009) [22] correlations for the HTC, homogenous flow-model correlations [13] for the pressure drop and with a cell size of $50 \mu \mathrm{m} \times 200 \mu \mathrm{m}$. The non-uniform power dissipation information was obtained by running var-
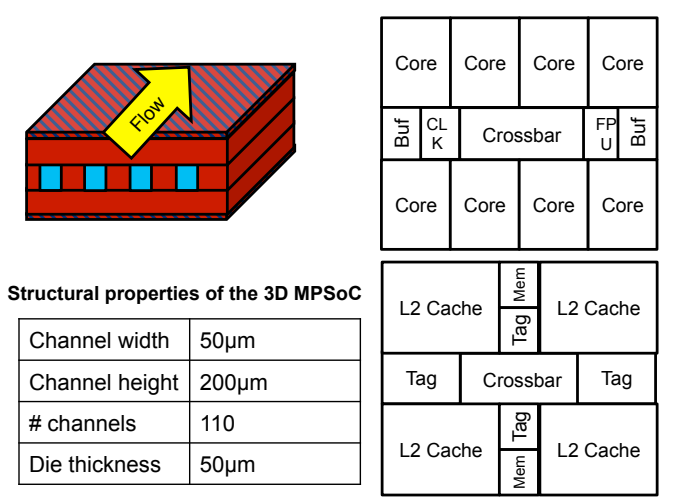

Figure 10: Structure and the floorplans of the 3D MPSoC simulated in Section 6 [15].

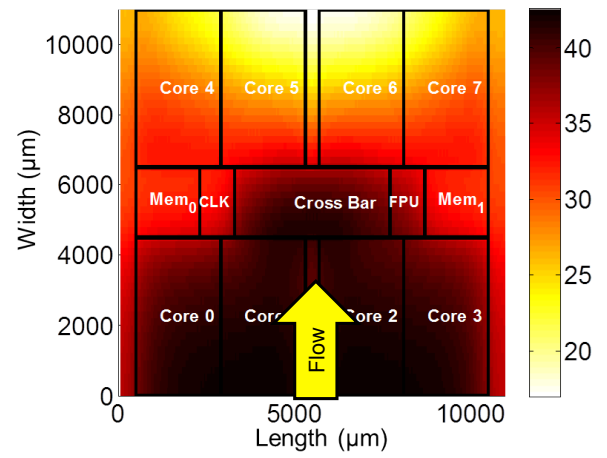

Figure 11: Thermal map of the core layer of the 3D MPSoC for the 'Web med' benchmark.

ious real-life benchmark applications on this architecture in an FPGA and obtaining the utilization values of each floorplan element in the 3D MPSoC [15].

For each benchmark, the simulation of the complete 3D MPSoC stack took only $54 \mathrm{sec}$, demonstrating the suitability of STEAM in early-stage design iterations. The resulting thermal map of the core layer for one of the benchmarks is shown in Fig. 11. As can be seen from the plot, temperatures near the inlet are higher than those near the outlet, which is counter-intuitive with respect to single-phase cooling [6]. But as explained in Section 2, this trend is expected in two-phase cooling where the temperature of the refrigerant actually decreases as it flows across the IC. These considerations, among others, would impact any thermal optimization methodologies to be developed for two-phase cooled MPSoCs in the future.

Based on the simulations, the total heat generated, the maximum temperature and the pumping power for each benchmark are tabulated in Table 3. The pumping power is calculated using the pressure drop calculations implicit in STEAM simulations (Section 4.2.2). Note that this represents purely the energy required to pump the refrigerant through the microchannel heat sinks. In a real two-phase cooled thermal package, there would be other important contributors to the total cooling energy cost, such as pump efficiency, chillers etc., discussions on which are beyond the scope of this paper. Nevertheless, the above experiments demonstrate the viability of STEAM as an effective tool to evaluate the thermal reliability and estimating the cooling costs of two-phase cooled 2D and 3D ICs. 
Table 3: STEAM simulation results for the test 3D MPSoC running various benchmark applications

\begin{tabular}{|c|c|c|c|}
\hline Benchmark & Total heat & Max Temp & Avg. Pumping Power \\
\hline Web med & $43.08 \mathrm{~W}$ & $42.55^{\circ} \mathrm{C}$ & $43.7 \mathrm{~mW}$ \\
\hline Web high & $54.77 \mathrm{~W}$ & $44.84^{\circ} \mathrm{C}$ & $55.5 \mathrm{~mW}$ \\
\hline Web DB & $51.37 \mathrm{~W}$ & $44.21^{\circ} \mathrm{C}$ & $53.6 \mathrm{~mW}$ \\
\hline Web low & $43.45 \mathrm{~W}$ & $42.63^{\circ} \mathrm{C}$ & $44.1 \mathrm{~mW}$ \\
\hline Database & $28.74 \mathrm{~W}$ & $43.87^{\circ} \mathrm{C}$ & $28.6 \mathrm{~mW}$ \\
\hline MPlayer & $21.58 \mathrm{~W}$ & $45.26^{\circ} \mathrm{C}$ & $21.6 \mathrm{~mW}$ \\
\hline MPlayer Web & $38.17 \mathrm{~W}$ & $42.31^{\circ} \mathrm{C}$ & $38.3 \mathrm{~mW}$ \\
\hline
\end{tabular}

\section{CONCLUSIONS}

In this paper we have presented a new thermal model called STEAM, which simulates the temperatures in an IC with two-phase cooling. The fundamentals of two-phase cooling and the derivation of the STEAM model from first principles have been described. The accuracy of the STEAM model has been validated against measurements from a real IC with two-phase cooling. Average errors incurred by the model have been found to be dependent on the particular heat transfer correlation studies employed in the model and can be as low as $10.2 \%$. Finally, we performed several simulation experiments on a realistic 3D MPSoC, demonstrating that STEAM can serve as a low complexity tool for earlystage temperature-aware design of ICs with two-phase cooling. The STEAM thermal model will be freely distributed to the research community soon at [24].

\section{Nomenclature}

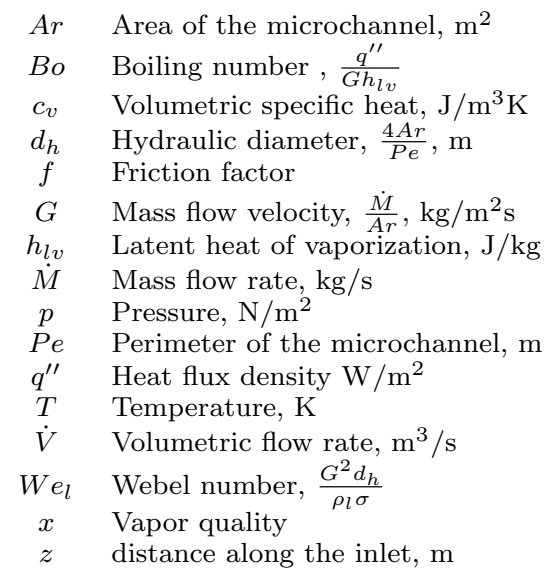

\section{Greek Symbols}

$\alpha$ Heat transfer coefficient, $\mathrm{W} / \mathrm{m}^{2} \mathrm{~K}$

$\mu$ Dynamic viscosity, $\mathrm{Ns} / \mathrm{m}^{2}$

$\rho$ Density, $\mathrm{kg} / \mathrm{m}^{3}$

$\sigma$ Surface tension, $\mathrm{N} / \mathrm{m}$

\section{Subscripts}

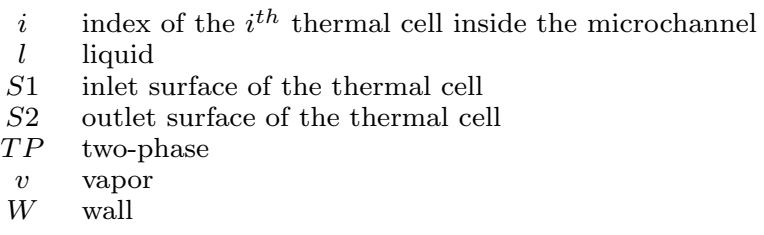

\section{REFERENCES}

[1] B. Agostini et al., "State of the art of high heat flux cooling technologies," Heat Transfer Engineering, vol. 28, no. 4, pp. 258-281, 2007.
[2] Y. Kim et al., "Thermal characterization of interlayer microfluidic cooling of three-dimensional integrated circuits with nonuniform heat flux," Journal of Heat Transfer, vol. 132, no. 4,2010 .

[3] T. Brunschwiler et al., "Heat-removal performance scaling of interlayer cooled chip stacks," in Thermal and Thermomechanical Phenomena in Electronic Systems (ITherm), 2010 12th IEEE Intersociety Conference on, june 2010, pp. $1-12$.

[4] B. Dang et al., "Integrated microfluidic cooling and interconnects for $2 \mathrm{~d}$ and $3 \mathrm{~d}$ chips," IEEE Transactions on Advanced Packaging, vol. 33, no. 1, pp. 79-87, 2010.

[5] F. Incropera et al., Fundamentals of heat and mass transfer. John Wiley and Sons, 2007.

[6] M. M. Sabry et al., "Thermal balancing of liquid-cooled 3d-mpsocs using channel modulation," in DATE, 2012.

[7] E. Costa-Patry et al., "On-chip two-phase cooling with refrigerant $85 \mu \mathrm{m}$-wide multi-microchannel evaporator under hot-spot conditions," Components, Packaging and Manufacturing Technology, IEEE Transactions on, vol. 2, no. 2, pp. $311-320$, feb. 2012.

[8] B. Agostini and M. Habert, "Compact thermosyphon heat exchanger for power electronics cooling," in 16th International Heat Pipe Conference, may 2012.

[9] I. Canturk, Workload Adaptive Power Management with Live Phase Monitoring and Prediction. Ph.D Thesis, Princeton University, June 2007.

[10] J. G. Collier and J. Thome, Convective Boiling and Condensation. Oxford Press, 1994.

[11] M. Moran et al., Fundamentals of Engineering Thermodynamics. John Wiley and Sons, 1988.

[12] M. Magnini et al., "Numerical investigation of hydrodynamics and heat transfer of elongated bubbles during flow boiling in a microchannel," International Journal of Heat and Mass Transfer, vol. 59, pp. 451-471, 2013.

[13] J. Thome, Wolverine Heat Transfer Engineering Data book III. [Online]. Available: http://www.wlv.com/ products/databook/db3/DataBookIII.pdf

[14] E. Costa-Patry et al., "On-chip two-phase cooling with refrigerant $85 \mu \mathrm{m}$-wide multi-microchannel evaporator under hot-spot conditions," Components, Packaging and Manufacturing Technology, IEEE Transactions on, vol. 2, no. 2 , pp. $311-320$, feb. 2012.

[15] M. M. Sabry et al., "Energy-Efficient Multi-Objective Thermal Control for Liquid-Cooled 3D Stacked Architectures," IEEE Transactions On CAD, vol. 30, no. 12, pp. 1883-1896, 2011.

[16] W. Huang et al., "Hotspot: a compact thermal modeling methodology for early-stage vlsi design," Very Large Scale Integration (VLSI) Systems, IEEE Transactions on, vol. 14, no. 5, pp. $501-513$, may 2006.

[17] A. Sridhar et al., "3D-ICE: Fast compact transient thermal modeling for 3D-ICs with inter-tier liquid cooling," in ICCAD, 2010, pp. 463-470.

[18] E. Costa-Patry et al., "Hot-spot self-cooling effects on two-phase flow of r245fa in $85 \mu \mathrm{m}$-wide multi-microchannels," in Thermal Investigations of ICs and Systems (THERMINIC), 2010 16th International Workshop on, oct. 2010, pp. 1 -6.

[19] "Intel single-chip cloud computer," http://www.intel.com/content/www/us/en/research/ intel-labs-single-chip-cloud-computer.html.

[20] T. Tran et al., "Small circular- and rectangular-channel boiling with two refrigerants," International Journal on Multiphase Flow, no. 3, pp. 485-498.

[21] NIST Standard Reference Database. [Online]. Available: http://www.nist.gov/srd/nist23.cfm

[22] S. Bertsch et al., "A composite heat transfer correlation for saturated flow boiling in small channels," Int. J. Heat Mass Transfer, vol. 52, 2009.

[23] S. Kandlikar and P. Balasubramanian, "Correlation to transition, laminar, and deep laminar flows in minichannels and microchannels," Heat Transfer Engineering, vol. 25, no. 3, 2004.

[24] A. Sridhar et al. http://esl.epfl.ch/STEAM. 